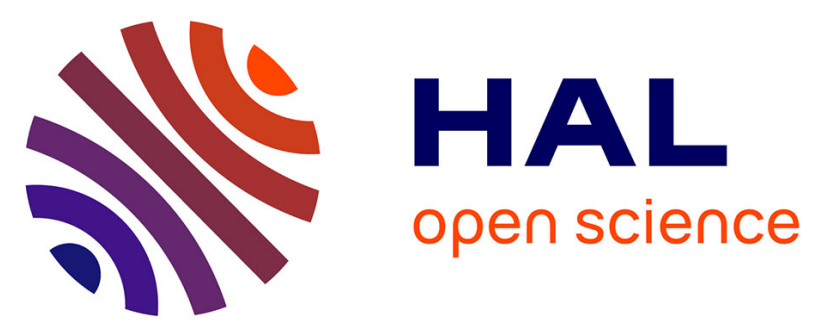

\title{
Une méta-analyse de récits de pratique de formatrices et de formateurs: l'élaboration d'un savoir collectif pour former le personnel enseignant à la déconstruction du genre
}

Gaël Pasquier, Gabrielle Richard

\section{To cite this version:}

Gaël Pasquier, Gabrielle Richard. Une méta-analyse de récits de pratique de formatrices et de formateurs: l'élaboration d'un savoir collectif pour former le personnel enseignant à la déconstruction du genre. Recherches féministes [revue interdisciplinaire francophone d'études féministes], 2018, 31 (1), pp.141 - 160. 10.7202/1050658ar . hal-02510507

\author{
HAL Id: hal-02510507 \\ https://hal.science/hal-02510507
}

Submitted on 17 Mar 2020

HAL is a multi-disciplinary open access archive for the deposit and dissemination of scientific research documents, whether they are published or not. The documents may come from teaching and research institutions in France or abroad, or from public or private research centers.
L'archive ouverte pluridisciplinaire HAL, est destinée au dépôt et à la diffusion de documents scientifiques de niveau recherche, publiés ou non, émanant des établissements d'enseignement et de recherche français ou étrangers, des laboratoires publics ou privés. 


\section{Une méta-analyse de récits de pratique de formatrices et de formateurs : l'élaboration d'un savoir collectif pour former le personnel enseignant à la déconstruction du genre}

\section{GAËL PASQUIER ET GABRIELLE RICHARD}

Depuis les années 70 et 80 , d'abord dans les pays anglo-saxons, puis en Europe francophone, des études mettent en évidence les parcours et les vécus scolaires différents des filles et des garçons. Trois types de constats révèlent l'existence de processus structurels de socialisation genrée touchant l'entièreté du milieu scolaire. Premièrement, les pratiques éducatives des enseignantes et des enseignants diffèrent en fonction du sexe de leurs élèves. Il y a davantage d'interaction avec les garçons; ceux-ci sont confrontés à des échanges plus complexes (Duru-Bellat 1995), et l'on se souvient plus promptement de leurs prénoms (Zaidman 1996). Les filles sont plutôt félicitées pour leur comportement (bonne conduite, travaux soignés) et blâmées sur la qualité intellectuelle de leur travail, alors que les observations inverses s'appliquent majoritairement aux garçons (Bressoux et Pansu 2003); ces derniers sont ainsi plus souvent l'objet de sanctions (Ayral 2011).

Deuxièmement, les contenus scolaires tant formels qu'informels présentent un caractère androcentré non négligeable et véhiculent des représentations réduites, stéréotypées et inégalitaires des femmes (Mosconi 1998; Baudoux et Noircent 1997). Troisièmement, les filles sont moins susceptibles de traduire sur le plan social et professionnel leur meilleure réussite scolaire (Bouchard et St-Amant 1996). Elles s'investissent majoritairement dans un nombre limité de filières, souvent moins prestigieuses. D'autres facteurs amènent à nuancer cette réussite pour certaines d'entre elles : même si les filles de parents ouvriers réussissent mieux que les garçons dans la même situation, "elles ne rattrapent pas les fils de cadre » (Bereni et autres 2012 : 148). Parallèlement, les garçons des milieux populaires, souvent moins familiarisés avec les attendus de l'école, sont davantage aux prises avec l'échec scolaire (Bonnery 2014). L'école accompagne donc autant qu'elle construit des expériences scolaires marquées par le système de genre qu'il importe de lire à l'intersection d'autres rapports sociaux.

Malgré ces constats récurrents sur les ambivalences de la mixité scolaire, la formation du personnel enseignant aux questions relatives à l'égalité des sexes a tardé à être mise en œuvre en Europe francophone (en France, ainsi qu'en Belgique et en Suisse francophones). Bien que des préconisations officielles existent, ces enseignements ne sont souvent inclus qu'avec parcimonie dans les programmes de formation initiale ou continue, où ils sont donnés en fonction des initiatives individuelles de formatrices ou de formateurs, de leur capacité à interpeller les 
PASQUIER ET RICHARD $\mid 142$

institutions, des compétences disponibles et de la bonne volonté des administrations. Afin de pallier l'isolement généré par la difficile institutionnalisation de ces initiatives, des formatrices et des formateurs ont cherché à échanger autour de leurs démarches de formation par l'écriture d'articles rendant compte de leur expérience et l'analysant. Ces récits de pratique deviennent l'occasion pour ces personnes de réfléchir autour des principales résistances rencontrées et des stratégies pédagogiques préconisées pour les contourner.

La méta-analyse élaborée dans le présent article propose une relecture critique de ces témoignages et de ces réflexions pour en souligner les apports au regard des enjeux actuels de formation en enseignement et interroger, à travers les démarches mises en œuvre, certains dilemmes ou tensions de la formation à l'égalité des sexes qu'ils mettent en avant.

\section{La formation des enseignantes et des enseignants à l'égalité des sexes en Europe francophone}

Les politiques éducatives en faveur de l'égalité des sexes en Belgique, en France et en Suisse s'inscrivent dans un cadre international et ont été légitimées par l'engagement de l'Organisation des Nations unies (ONU) et de l'Europe, avant de donner lieu à des traductions ou à des initiatives locales qui témoignent d'enjeux spécifiques (Pasquier 2013b; ANEF 2014). Il a toutefois fallu attendre l'année 1999 pour qu'un avis du Conseil de l'éducation et de la formation de la Communauté française de Belgique recommande de former le personnel enseignant à l'égalité des sexes, et l'année 2005 pour que « la dimension de genre» soit effectivement inscrite dans le décret organisant la formation initiale pour l'éducation préscolaire, le primaire et le premier cycle du secondaire. C'est essentiellement le mouvement associatif féministe qui a porté les questions relatives à l'égalité filles-garçons et qui est à l'origine des premières formations mises en œuvre dans les hautes écoles responsables de la formation du personnel enseignant (Plateau 2007 et 2014). D'une manière générale, il apparaît que cette formation a tardé et tarde encore à se développer.

En Suisse, où le système d'enseignement est organisé par cantons, la Conférence des directeurs de l'instruction publique recommande de former l'ensemble des enseignantes et des enseignants à la question de l'égalité des sexes depuis 1993. Ses avis ne sont toutefois pas contraignants et « les questions liées au genre sont actuellement peu développées dans la formation à l'enseignement » (Jacquemet 2013 : 77). Elles y font leur apparition en 2007 pour le canton de Genève qui semble faire figure d'exception. Cette introduction s'est traduite par une véritable réflexion pédagogique sur les modalités d'intervention choisies et par l'intention de prendre en considération le genre « dans tous les aspects de la formation, didactiques ou transversaux » (Collet et Grin 2013b : 35). En 2009, un module intitulé « Genre et éducation » est rendu obligatoire pour les enseignantes et les enseignants du secondaire; en 2012, pour celles et ceux du primaire. Dans les autres cantons 
UNE MÉTA-ANALYSE DE RÉCITS DE PRATIQUE $\mid 143$

francophones, certains enseignements sont mis en place au sein des hautes écoles pédagogiques (HEP), mais leur institutionnalisation reste fragile à ce jour.

En France, malgré la récurrence des constats et la publication régulière de textes institutionnels en faveur de l'égalité des sexes à l'école, la formation des enseignants et des enseignantes demeure l'un des points obscurs des politiques d'égalité. Le Cahier des charges de la deuxième année de formation dans les instituts universitaires de formation des maîtres (IUFM), conçu par le ministère de l'Éducation nationale en 2002, stipule la nécessité de sensibiliser les futurs membres du personnel enseignant aux « représentations sociales, familiales, professionnelles de la femme et ses conséquences (choix de parcours, métiers) » et à « la gestion de la mixité scolaire » (non paginé). Il revient néanmoins à chaque IUFM de choisir la place et le volume horaire qui seront attribués à cet enseignement; de fait, il reste souvent optionnel, voire inexistant, à l'exception d'un intitulé dans le descriptif de certains modules. En 2013, l'arrêté qui fixe le cadre national des formations précise qu'elles doivent comprendre un enseignement lié aux «principes et à l'éthique du métier, dont [...] la lutte contre les discriminations et la culture de l'égalité entre les femmes et les hommes ». Néanmoins, comme l'ont relevé deux rapports récents (Dhume, Sotto et El Massioui 2015; HCE 2016), «l'offre de formation est aujourd'hui incomplète et disparate sur l'ensemble du territoire [...] : seule la moitié des ESPE [écoles supérieures du professorat et de l'éducation] considère avoir formé la totalité de leurs étudiant.e.s avec un volume horaire variant entre 2 heures et 57 heures annuelles. Seule la moitié des ESPE propose un module dédié à l'égalité filles-garçons » (HCE $2016: 6)^{1}$.

Cette situation rend les récits de pratique de formation d'autant plus précieux qu'ils permettent de documenter la situation, tant sur le plan des contextes institutionnels que sur celui des objectifs, des contenus et des pratiques pédagogiques mis en œuvre. Ils constituent par ailleurs des ressources pour les formatrices et les formateurs qui souhaiteraient approfondir une telle question.

\section{Les démarches méthodologiques et la présentation des récits de pratique}

Notre article prend appui sur 33 récits de pratique rédigés par des formatrices et un formateur engagés dans la sensibilisation aux inégalités genrées de futurs membres du personnel enseignant, et ce, de la maternelle/éducation préscolaire à l'enseignement secondaire, en formation initiale ou continue. Par « récit de pratique », nous entendons la narration par les auteures et auteurs de leur propre démarche pédagogique (ou de celle de leurs collègues) et des réflexions sur cette pratique. Celles-ci s'ancrent à différents degrés dans des réflexions théoriques : cette dimension

1 L'Association de recherche sur le genre en éducation et formation (ARGEF) a élaboré en 2017, en collaboration avec l'Association nationale des études féministes (ANEF), une carte des enseignements à l'égalité femmes-hommes dans les ESPE de France : www.argef.org/carte-de-france-des-espe/ (27 juillet 2017). 
PASQUIER ET RICHARD | 144

autoréflexive paraît en effet centrale pour les auteures et les auteurs de ces récits et participe systématiquement de leur projet.

Les récits de pratique ont été repérés sur des moteurs de recherche d'articles scientifiques (Google Scholar, Isidore) et des ressources en libre accès (Jurn), en croisant les mots clés formation, éducation et apprentissage avec sexisme, égalité des sexes, égalité filles garçons, inégalité de genre et rapport sociaux de sexe; à cela s'est ajoutée notre propre connaissance de la littérature dans ce domaine. Un tri subséquent nous a permis de sélectionner les articles correspondant à des récits de pratique, publiés depuis 2000 en Europe francophone. Toutefois, il ne semble pas que de tels récits aient été publiés avant 2005. Le récit de Lambert est tiré de notes prises lors d'une communication; nous l'avons maintenu dans l'échantillon parce qu'il nous a paru témoigner d'un positionnement singulier. La liste exhaustive des récits composant le corpus se trouve en fin d'article.

L'analyse du corpus s'est faite à partir de la méthode d'analyse inductive générale telle que l'ont décrite Mireille Blais et Stéphane Martineau (2006). Elle a consisté à relire à plusieurs reprises les récits de pratique retenus et à en rédiger des résumés succincts, de manière à nous en approprier le contenu. La dernière de ces relectures, directement guidée par les objectifs de la recherche, nous a permis de déterminer des catégories, tout en assurant une vigilance à la cooccurrence de thématiques émergeant directement du corpus. À l'aide d'un tableau Excel, nous avons systématisé nos observations en quatre catégories (formation développée ou offerte; démarche mise en œuvre; dilemmes et tensions; apports relatifs à l'égalité hommes-femmes), elles-mêmes subdivisées en fonction du niveau de détail offert par les récits. Nous avons réalisé individuellement ces exercices de codage en parallèle, de manière à nous assurer de la meilleure représentation du corpus au vu de la problématique étudiée.

L'essentiel des récits répertoriés ont été produits par des chercheuses françaises, dont la majorité sont affiliées à des IUFM ou à leurs incarnations plus récentes, les ESPE. La plupart exercent en France, d'autres (également) en Suisse francophone (Isabelle Collet, Céline Petrovic, Nicole Jacquemet, Nadia Lamamra et Myriam Posse). Une seule formatrice travaille en Belgique francophone (Nadine Plateau).

Les auteures et l'auteur que nous avons étudiés s'inscrivent dans deux types de démarches. La plupart proposent une réflexion basée sur la formation. Les récits de pratique sont alors l'occasion de porter un regard critique sur leur démarche pédagogique et sur ses impacts. Cela se donne particulièrement à voir chez Céline Petrovic, dont les récits successifs se lisent comme autant d'étapes d'affinement de ses pratiques de formation. Le récit peut être aussi l'occasion de travailler à dénouer ce qui a été perçu comme une impasse ou vécu comme une difficulté (Plateau 2007). D'autres praticiennes et praticiens conjuguent formation et recherche, et sondent leurs stagiaires afin de déterminer les apprentissages maîtrisés ou rejetés au terme de la formation (Collet et Grin 2013a; Lamamra et Posse 2013), ou dans l'objectif de mieux 
UNE MÉTA-ANALYSE DE RÉCITS DE PRATIQUE | 145

comprendre les biais genrés que ces stagiaires peuvent parfois entretenir sans le savoir (Morin-Messabel, Ferrière et Salle 2012).

\section{Les résistances aux apprentissages sur le genre et l'adaptation conséquente de la formation}

Plusieurs récits semblent avoir été écrits pour « faire face à une solitude et un isolement dangereux » (Baurens 2005 : 135). Ce besoin tient en partie au contexte dans lequel ont été élaborées les formations, sans véritable soutien institutionnel. Il semble moins formulé dans les textes les plus récents, bien que ceux-ci continuent à être motivés par l'intention de constituer une communauté de réflexion pour mutualiser les pratiques mises en œuvre, notamment en ce qui concerne les résistances rencontrées, et comparer les approches. Les auteures et l'auteur de ces différents récits se lisent en effet pour la plupart mutuellement, comme en témoignent les bibliographies des textes examinés et les dialogues établis entre certains textes.

Quels que soient les contextes sociopolitiques au sein desquels elles sont élaborées, les formations dont ces récits de pratique rendent compte visent deux objectifs. Le premier consiste à sensibiliser les stagiaires aux inégalités filles-garçons et aux multiples manières dont elles peuvent se manifester en milieu scolaire (répartition des élèves, orientation scolaire, représentations dans les manuels, violences de genre, etc.). Le second objectif est de les amener à interroger leur propre rôle dans la production et la reproduction d'expériences scolaires inégalitaires basées sur le sexe. L'enjeu ultime est la construction, la transformation ou l'adaptation de leurs pratiques pédagogiques pour qu'elles soient davantage porteuses d'égalité ${ }^{2}$. Ce faisant, ce qui se trouve véritablement au cœur du projet est «la capacité des personnes à se confronter à leur propre participation au système de genre, à questionner leur position dans les rapports sociaux de sexe (et également dans les rapports sociaux entre enseignant-e et enseigné-e-s) et à leur capacité à vouloir intervenir sur ce système » (Lamamra et Posse 2013 : 74).

\section{Des récits centrés sur les résistances}

L'un des constats de notre recension concerne la centration de plusieurs récits de formation sur l'existence de résistances. L'égalité des sexes et les rapports sociaux

2 Conjointement à des évènements comme les journées d'étude Genre et didactique des disciplines organisées à l'ESPE de Créteil en 2014, l'ouvrage d'Annie Lechenet, de Mireille Baurens et d'Isabelle Collet (2016) - par l'entremise des récits de Nathalie Sayac (2016) et de Vincent Porhel (2016) qui s'intéressent respectivement à l'infusion d'une approche genrée en didactique des mathématiques et en histoire - semble participer d'une inflexion d'une approche transversale vers des approches consacrées précisément à la question des savoirs scolaires et de leur construction, ainsi qu'aux didactiques des disciplines dans une perspective genrée. 
PASQUIER ET RICHARD | 146

de sexe constituent en effet des sujets d'enseignement sensibles et présentent un potentiel déstabilisateur fort, au même titre que d'autres thématiques évoquant l'oppression, les rapports de pouvoir, les privilèges et les identités (Richard à paraître). Il est toutefois permis de penser que le caractère optionnel de la majorité des formations actuelles engendre une sélection de l'effectif étudiant en faveur de celles et ceux qui sont déjà sensibilisés à ce sujet (ou minimalement ouverts à la discussion), ce qui permet d'éviter les formes d'opposition les plus frontales.

\section{Les résistances des stagiaires mises au centre de la réflexion sur la formation}

En d'autres termes, les récits peuvent être perçus comme autant de quêtes d'une bonne ou meilleure approche pédagogique, laquelle serait garante des impacts positifs de la formation. C'est du moins l'hypothèse sous-jacente dans tous les récits, à l'exception de celui de Karine Lambert, formatrice à l'ESPE de l'Académie de Nice, qui souligne plutôt les résistances des corps d'inspection et des chefs d'établissement. Devant le manque de temps accordé à la thématique du genre en éducation, à la quête constante de légitimité politique, celle-ci dresse un constat d'échec. Son récit fait toutefois exception. L'essentiel des autres textes s'articulent autour de trois types de questions :

- Quelles sont les pratiques mises en place?

- Quels succès et quelles limites présentent-elles?

- Comment peut-on améliorer ces pratiques de manière à générer une prise de conscience des stagiaires, voire une transformation de leurs pratiques éducatives?

Trois grandes certitudes à ébranler apparaissent comme centrales : l'« égalité déjà-là ", l'idéologie féministe et l'intervention impossible. Le mythe de l'égalité « déjà-là » (Delphy 2007), première certitude, suggère que l'égalité entre femmes et hommes serait atteinte, et que les revendications féministes seraient en décalage par rapport à la réalité des rapports entre les sexes. Dans les formations, cette certitude de l'égalité déjà atteinte s'observe à trois niveaux. Des stagiaires peuvent considérer que l'égalité des sexes est socialement atteinte et, par conséquent, contester les savoirs attestant le contraire (voir, par exemple, Petrovic (2013a et 2013b) et Baurens (2008)). D'autres conviennent que certains combats restent à gagner (égalité salariale, accès à des postes de pouvoir, etc.), mais pensent que le système scolaire est globalement exempt de ces inégalités (voir, par exemple, Jacquemet (2013), Baurens et Schreiber (2010) et Costes (2014)). Finalement, des stagiaires estiment que, si la société et l'école véhiculent des inégalités structurelles, ces dernières n'ont pas de prise sur la manière dont, en tant qu'enseignantes ou enseignants, elles et ils interagissent avec leurs élèves (Collet et Grin 2013b; Morin-Messabel, Ferrière et Salle 2012; Baurens 2008). Si des différences de traitement sont constatées entre les sexes, les stagiaires ont tôt fait de suggérer qu'elles résultent davantage de la famille et des médias que de l'école. 
UNE MÉTA-ANALYSE DE RÉCITS DE PRATIQUE | 147

D'après les formatrices et les formateurs, les apprentissages sur le genre seraient perçus par beaucoup de stagiaires comme des menaces à l'ordre établi, tant sur le plan de leur identité professionnelle que sur celui de leurs croyances personnelles sur les rapports entre les sexes et la manière dont la société fonctionne. Ainsi, non seulement on leur explique que dans les faits l'école n'est pas égalitaire, mais les enseignantes et les enseignants en formation sont en plus placés devant la possibilité de participer activement à la reconduction de normes genrées dommageables pour les élèves. La suggestion que leur propre attitude puisse être la courroie de transmission de ces inégalités peut occasionner un refus d'entendre, voire de l'agressivité, chez des personnes, convaincues de n'opérer aucune distinction entre les filles et les garçons (Morin-Messabel, Ferrière et Salle 2012).

La deuxième certitude à ébranler concerne l'affirmation selon laquelle la formation ne serait pas scientifiquement fondée (Costes 2014), mais plutôt alimentée par une idéologie féministe (Collet et Grin 2013b) qui refuserait de dire son nom. Ce constat d'un refus à différents degrés d'accorder de la légitimité à la formation se donne à voir par le discrédit dont font l'objet ces apprentissages. Ainsi, on refuse d'accorder quelque validité que ce soit aux données et aux statistiques présentées, suggérant qu'elles relèvent davantage d'un programme féministe que d'une démarche scientifique valide (Fontanini 2005). Le contexte sociopolitique français a certainement contribué à ce que cette certitude gagne en popularité depuis 2011, année où commence une campagne de désinformation sur les enseignements sur le genre à l'école nourrie par le spectre d'une "théorie du genre " cherchant à favoriser l'indifférenciation sexuelle (Salle 2014) ${ }^{3}$.

L'étiquetage idéologique de la formation à l'égalité des sexes peut également s'expliquer par le fait qu'elle oblige les stagiaires à penser leur propre positionnement dans les rapports sociaux de sexe. Les hommes se trouvent ainsi à devoir affronter des représentations collectives de la masculinité axées sur la domination et le privilège dans lesquelles ils peuvent ne pas se reconnaître en tant qu'individus. Les femmes, pour leur part, sont amenées à considérer qu'elles sont plus susceptibles d'être violentées ou payées en deçà de leur valeur du seul fait d'être nées femmes ${ }^{4}$. La primauté du sexe biologique en tant que marqueur principal du positionnement social n'est donc pas sans conséquence sur l'image de soi.

La troisième certitude freinant les stagiaires est qu'il se révèle difficile, voire impossible, d'agir en tant qu'enseignantes ou enseignants sur les normes du genre de manière à avoir un impact sur les rapports de pouvoir en jeu, en raison notamment $d u$

3 À l'inverse, il est aussi possible que ce contexte ait joué un rôle de sensibilisation des étudiantes et des étudiants en formation en diffusant des savoirs auxquels on sera d'autant plus sensible qu'ils bénéficient davantage de visibilité.

4 Les unes et les autres ont probablement eu, bien qu'aucun récit n'insiste explicitement sur cet aspect, à relire le parcours qui les a menés à vouloir entrer dans l'enseignement, profession aujourd'hui encore majoritairement exercée par des femmes, sous un jour sans doute plus inconfortable. 
PASQUIER ET RICHARD $\mid 148$

nombre d'instances de socialisation qui participent à leur reproduction. Le passage à l'action des stagiaires, au terme de la formation, est une préoccupation qui revient dans bon nombre de récits de pratique (Baurens et Schreiber 2010; Couchot-Schiex, Cogérino et Coltice 2009). Plusieurs attendent en outre que leur soient fournis les outils permettant la mise en œuvre de l'égalité filles-garçons par des interventions simples (Lamamra et Posse 2013). Or, la transformation des pratiques des stagiaires est tout sauf acquise au terme d'un module de formation de quelques heures.

Ces trois certitudes correspondent à des blocages qui peuvent intervenir à différentes étapes du processus de formation. Et il semble nécessaire aux auteures et aux auteurs de faire face à ces blocages lorsqu'ils se produisent pour éviter qu'ils n'entraînent chez celles et ceux qui les vivent différentes postures de fermeture, des crispations ou le sentiment d'une menace (lequel peut s'incarner par des réactions vives, de l'obstruction, un monopole de la parole, etc.) (voir, par exemple, Lamamra et Posse (2013) et Costes (2014)).

\section{Des dispositifs pour ébranler les résistances}

Les pistes de réflexion élaborées dans les récits de pratique dans l'objectif de troubler les résistances sont nombreuses. Elles peuvent autant porter sur un aspect de la formation (en suggérant de clarifier en amont des règles de bienveillance encadrant les interactions) que sur l'entière posture parrainant l'intervention. Ces propositions ont en commun le souci de prouver la pertinence de parler du genre et des rapports sociaux de sexe, de confirmer la légitimité à le faire dans le contexte de la formation du personnel enseignant, ainsi que de soutenir les stagiaires dans des apprentissages susceptibles d'être considérés comme sensibles ou déstabilisants.

\section{L’égalité « déjà-là »}

La littérature scientifique est largement mobilisée dans les formations pour démontrer, de manière empiriquement fondée et statistiquement soutenue, que les inégalités entre les sexes demeurent des problématiques réelles qui méritent d'être analysées. L'indice de difficulté est majeur pour les formatrices et les formateurs qui doivent s'emparer de traditions disciplinaires variées et manier des notions parfois complexes, souvent éloignées de leur champ d'expertise : la neurobiologie pour évoquer la plasticité du cerveau, la linguistique pour discuter la construction genrée de la langue française, l'économie et la sociologie pour expliquer les écarts salariaux ou la division des tâches au sein du couple, etc. Mireille Baurens (2008) propose par ailleurs le développement d'une compétence transculturelle du genre, suggérant que la prise de conscience des inégalités à un niveau local serait favorisée par l'évocation d'autres cadres normatifs dans d'autres époques ou d'autres lieux géographiques.

C'est toutefois le regard que les stagiaires posent sur leurs propres perceptions qui semble davantage préoccuper les formatrices et les formateurs qui cherchent à 
UNE MÉTA-ANALYSE DE RÉCITS DE PRATIQUE | 149

démontrer aux stagiaires qu'elles et ils véhiculent bien des stéréotypes de sexe, même si, à leur avis, ce n'est pas le cas. Des formatrices suggèrent qu'un temps d'échange et de partage d'expériences entre stagiaires est nécessaire pour permettre l'amorce de cette autoréflexion au cours du module de formation (Guilpain 2016; Petrovic 2013b). D'autres estiment que cette prise de conscience se fera suite à des évènements déclencheurs (un incident critique, l'observation d'un ou d'une collègue), pendant leur stage ou après leur entrée en poste (Couchot-Schiex, Cogérino et Coltice 2009).

Christine Morin-Messabel, Séverine Ferrière et Muriel Salle (2012) se livrent avec leurs stagiaires à un exercice destiné à leur faire prendre conscience de ces stéréotypes. Il leur est demandé d'évaluer un ou une élève de CM2 (élève de 1011 ans; cinquième année du primaire dans le système scolaire québécois) qui réussit bien dans toutes les disciplines, sauf en mathématiques. Les formatrices font remarquer à leurs stagiaires que leurs évaluations diffèrent selon que le nom donné à l'élève est Sarah ou Adam. Elles espèrent que cette confrontation à leurs propres représentations fera émerger chez les stagiaires une réflexivité sur leurs pratiques. Des démarches similaires d'observation et d'analyse des comportements et des attitudes sont reprises dans d'autres cadres par les formatrices et les formateurs accompagnant leurs stagiaires dans la réalisation d'un mémoire professionnel ou d'initiation à la recherche (Nicole Jacquemet, Vincent Porhel, Nathalie Sayac, Nadia Lamamra et Myriam Posse).

\section{L’idéologie féministe}

L'une des principales résistances mises en évidence est la faible légitimité accordée par les stagiaires à une formation sur le genre en éducation, dans un contexte où les études de genre souffrent de discrédit social et universitaire (Trachman 2014). La découverte de ce frein par certaines formatrices leur a permis de modifier des parties de la formation dans l'objectif de signifier clairement que leur démarche ne résulte pas d'un programme féministe ou de l'initiative isolée de personnes militantes, mais bien d'efforts concertés de l'institution, et appuyés à différents degrés par les instances gouvernementales ${ }^{5}$. Pour ce faire, elles sont nombreuses à mobiliser des textes de lois ou des dispositifs institutionnels et à rappeler que leurs séances s'inscrivent dans ce contexte (voir, par exemple, Costes (2014), Petrovic (2006, 2013a et $2013 b$ ) et Houadec (2016)).

Une stratégie détournée consiste à accorder davantage de temps de parole aux stagiaires (Petrovic 2013a et 2013b; Baurens et Schreiber 2010; Guilpain 2016). Cela permet d'offrir à certaines personnes la possibilité de verbaliser leur désaccord, et à d'autres, de présenter des contre-arguments, ce qui évite ainsi aux formatrices et aux formateurs de porter individuellement tout le poids de l'argumentation. Cette méthode

5 Selon nous, ce constat n'est pas propre à la France, mais concerne également la Belgique et la Suisse. 
PASQUIER ET RICHARD | 150

présente également le mérite d'ancrer les connaissances acquises dans les expériences de terrain (et d'ainsi montrer qu'il y a bien là matière à une problématique) et de favoriser un climat de coopération et de confiance entre les stagiaires. Bien entendu, le bon fonctionnement de ce mode pédagogique est conditionné au respect de règles de bienveillance et de confidentialité établies d'entrée de jeu et rappelées au besoin. Il nécessite également le maintien d'un juste équilibre entre la transmission d'informations en vue de couper court à l'expression de certaines résistances anticipées et la marge de manœuvre permettant aux stagiaires de les exprimer pour les penser collectivement.

De légères adaptations de langage peuvent également permettre de désamorcer certaines tensions ou d'éviter qu'elles se créent (Baurens et Schreiber 2010; Costes 2014; Petrovic 2006). Souligner qu'une part de libre arbitre demeure malgré les déterminants sociaux, ou encore clarifier que «les filles » constitue bel et bien une généralisation et ne signifie en aucun cas que toute fille agit d'une manière donnée, sont autant de précautions qui gagnent à être spécifiées pour éviter les accusations de parti pris féministe et les replis sur soi.

\section{L'intervention impossible}

Le passage de la théorie à l'action pose problème à une forte proportion de stagiaires ${ }^{6}$. Là encore, les récits de pratique convoquent des méthodes variées : rencontres avec des professionnelles ou des professionnels déjà à l'œuvre dans des établissements scolaires (Couchot-Schiex à paraître) ou encore invitation à la réflexion par l'entremise d'un questionnaire d'enquête (Baurens et Schreiber 2010) ou par la rédaction d'un mémoire (Jacquemet 2013). Dominique Gauthiez-Rieucau (2016 : 269) encourage l'engagement personnel des stagiaires et l'émergence de leurs représentations en leur proposant un travail de production littéraire pour lequel « le truchement du personnage facilite l'introspection et l'auto-critique, en termes de construction identitaire et de gestes professionnels $»$. Il s'agit donc, dans tous les cas, de contourner la perception de non-applicabilité des constats d'inégalité, de manière à engager les stagiaires dans la conception de leurs propres interventions.

6 C'est d'ailleurs sur le passage du questionnement à la modification des pratiques que se situe l'un des axes de travail encore à explorer. Si les pratiques en faveur de l'égalité des sexes et des sexualités commencent à être documentées (Pasquier 2013b; Richard 2014; Pasquier, Marro et Breton 2016; Marro, Pasquier et Breton 2016), si des outils transversaux ou disciplinaires existent, il reste nécessaire que «la recherche action s'oriente vers les descriptifs et l'analyse de leur mise en œuvre» (Guilpain $2016: 218$ ). 
UNE MÉTA-ANALYSE DE RÉCITS DE PRATIQUE $\mid 151$

D'un modèle pédagogique à l'autre

\section{Une prise en considération de l'intime (nécessairement) ambivalente?}

Si les formations dont nous avons parlé cherchent à interroger, et parfois même ébranlent, les représentations des stagiaires sur le plan tant professionnel que personnel, il n'en reste pas moins que ces dimensions ne sont pas prises en considération de la même manière par les formatrices et les formateurs. Les récits reconnaissent unanimement que le travail sur le genre interpelle la manière dont les personnes formées se sont construites en tant que femme ou homme, leur conception d'une vie sentimentale et sexuelle réussie, d'une éducation réussie, que ce soit celle de leurs enfants ou de leurs élèves. Ce travail met donc à mal la partition classique entre public et privé telle que l'ont remise en cause les mouvements féministes, et ce, dans un contexte professionnel. Les récits interrogent ainsi la possibilité de prendre en considération des éléments relevant de l'intime et du personnel dans un cadre de formation institutionnelle qui n'y accorde habituellement pas de place. Des formatrices disent ne pas (pouvoir) tenir compte de ces aspects, en raison du temps imparti et «se limite[r] délibérément à travailler l'identité professionnelle » en insistant « sur le fait que nous travaillons une question de métier » (Guilpain 2016 : 211). Cette stratégie n'empêche pas toujours ces questions de faire irruption dans le groupe. Surtout, elle interroge la responsabilité d'une institution qui bouscule les représentations personnelles de ses (futurs) salariés et salariées, sur une dimension structurante de leur identité psychique et sociale (Petrovic 2013a), mais n'est pas en mesure d'accompagner pleinement leur cheminement et de leur offrir un espace de travail pour ce faire. D'autant que ces questionnements personnels ne sont parfois pas sans mobiliser des enjeux liés au métier (Lambert 2015 : s. p.) :

Nos bureaux deviennent souvent un lieu de confidences. Je pense à beaucoup de jeunes enseignants garçons du premier degré qui s'interrogent beaucoup $\mathrm{du}$ fait de leur sexe et/ou de leur orientation sexuelle - sur leur place, leur légitimité et les dangers qu'ils courent, notamment lorsqu'ils ont des activités militantes publiques.

D'autres formatrices mettent en œuvre des dispositifs qui ont précisément pour objet de travailler ces dimensions et d'ouvrir des espaces de parole où certaines choses pourront être dites, écoutées et discutées, en veillant à ce que les situations mises en œuvre ne soient pas intrusives. Les cadres instaurés ne sont cependant pas toujours sans ambivalence : Mireille Baurens et Caroline Schreiber (2010: 82) disent ainsi commencer leurs formations par un remue-méninges (brainstorming) destiné à faire ressortir la «multiplicité des expériences" des stagiaires, mais c'est bien à la réinstauration d'une frontière public/privé qu'elles recourent en argument d'autorité lorsqu'à leurs yeux « certaines réactances [proviennent] d'une confusion entre le rôle 
PASQUIER ET RICHARD | 152

de parent et celui d'enseignant » (ibid. : 83). La situation semble ainsi jouer sur les deux tableaux. Et c'est bien toute la difficulté à laquelle semblent devoir faire face les formatrices et les formateurs qui cherchent à construire des spécialistes de la pratique réflexive mais qui, de par leur fonction, peuvent avoir paradoxalement recours à des injonctions institutionnelles supposées couper court temporairement à la contestation. Cette tâche est d'autant moins aisée que sous l'apparente continuité des textes officiels se révèle surtout l'embarras d'une institution à l'égard des enjeux liés au genre et véhiculant, notamment pour la France, des représentations concurrentes et parfois contradictoires de l'égalité en vue d'éviter toute polémique (Salle 2016; Pasquier à paraître).

\section{Quelle est la construction de compétences et de connaissances des stagiaires?}

Le travail sur les représentations mis en œuvre dans le contexte des formations amène les formatrices et les formateurs à octroyer le plus souvent une place importante aux échanges entre stagiaires. Une telle stratégie permet de partir de leurs représentations et expériences, parfois très hétérogènes, pour en faire des instruments de mobilisation, de questionnement et de problématisation. Elle offre en outre l'avantage « de créer un climat de coopération » qui « décharge [les formatrices] de [leur] statut de contradictrices perçues dans un lien hiérarchique » (Baurens et Schreiber $2010: 82$ ). Utiles, ces discussions où des éléments sont énoncés, des anecdotes racontées, pour être travaillés immédiatement ou repris plus tardivement, posent la question de ce qui est véritablement construit par les stagiaires. Ce cadre se révèle parfois déstabilisant pour des étudiantes et des étudiants habitués à des interventions magistrales ou à des cours dialogués qui continuent à reposer sur une relation frontale entre la personne qui enseigne et les élèves suscitant assez peu les relations entre élèves. Il donne à certaines personnes l'impression de n'avoir rien appris dans un contexte qui manque de rigueur (Richard à paraître). Et de fait, malgré les apports théoriques au fil des échanges et les références à des recherches destinées à renforcer le propos des formatrices et des formateurs, la discussion peut sembler parfois reposer sur l'énoncé par les stagiaires de représentations de sens commun qui sont ensuite mises en questions pour être remplacées par d'autres représentations de sens commun véhiculant cette fois-ci une conception qualifiée d'égalitaire des femmes et des hommes, et parfois des sexualités. Les apports de connaissances ne sont pas toujours perçus ni reconnus comme tels ou apparaissent trop furtivement pour que les stagiaires puissent véritablement s'en saisir. Le cadre participatif instauré peut alors susciter des «malentendus » (Bautier et Rayou 2009), entre les intentions des formatrices et des formateurs et ce que les stagiaires en perçoivent, en donnant l'impression qu'il suffit de discuter entre soi pour atteindre les objectifs fixés. Même lorsque ces temps d'échange ne sont pas dénigrés, ces malentendus peuvent parfois reposer sur des représentations antagonistes des rapports entre théorie et pratique, qui 
UNE MÉTA-ANALYSE DE RÉCITS DE PRATIQUE | 153

postulent l'inutilité de la seconde pour penser la première, et perçoivent dans le dispositif mis en œuvre une confirmation de ce présupposé : «ce qui les captive, ce sont les histoires et les anecdotes, ce qu'elles apprécient, ce sont les débats où elles peuvent s'exprimer » (Plateau 2007 : 102).

Ces retours sont particulièrement déstabilisants pour certaines formatrices qui, dans leur parcours féministe, ont participé à la construction d'un savoir collectif par des femmes dans des groupes de prises de conscience (Plateau 2007). Certes, elles entendent doter les stagiaires d'un savoir, ou à tout le moins d'outils, pour « décoder le vécu et y repérer les inégalités » (ibid. : 98), mais force est de constater que ce n'est pas la totalité des stagiaires qui réussit à saisir les éléments en jeu dans les situations proposées.

\section{Quelle pédagogie féministe peut-on favoriser en contexte institutionnel de formation du personnel enseignant?}

La possibilité d'explorer d'autres approches pédagogiques dans un contexte de formation initiale et continue des enseignantes et des enseignants où le public est captif et peu sensibilisé à d'autres modalités d'enseignements se lit donc en filigrane des récits étudiés. Plusieurs formatrices pointent en effet le caractère inapproprié des cours magistraux et recherchent des dispositifs participatifs et collaboratifs davantage conformes aux savoirs et aux compétences qu'elles entendent transmettre. Certaines semblent s'appuyer dans leur démarche sur les principes d'une pédagogie féministe, considérant les «multiples facettes » du savoir " qui n'est pas uniquement rationnel et constitue une expression [et une production] du pouvoir » (Ollagnier $2014: 66$ ), en insistant sur la contextualisation des apprentissages ou l'importance de la subjectivité dans la construction des connaissances. Elles recourent également parfois à des pratiques et à des modalités de communication coopératives en vue d'une coconstruction des savoirs. Un bon nombre disent enfin chercher à mettre en œuvre des rapports plus égalitaires entre celle ou celui qui forme et les personnes formées pour remettre en question la dimension hiérarchique et transmissive de la relation pédagogique entre une personne supposée savoir et d'autres devant apprendre. Leurs récits témoignent d'ailleurs bien souvent de cette approche par leurs réflexions sur les réussites et les échecs des formations, et mettent en acte, au-delà d'une simple proclamation de principes, la conviction que les formatrices et les formateurs ont aussi à apprendre de leurs stagiaires.

Il n'en reste pas moins que cette mise en œuvre n'est pas évidente et n'est pas elle non plus sans occasionner des résistances... à la pédagogie féministe ${ }^{7}$. D'une part,

7 Le rapport réalisé par Fabrice Dhume, Floréal Sotto et Nora El Massioui (2015: 86) nuance tout de même le tableau dressé : « Les observations de cours, notamment dans deux ESPE, montrent à l'envi des formatrices.teurs qui témoignent du souci de casser certains codes scolaires (par exemple, des tables en " $U$ " plutôt que le traditionnel format en " wagon "), de centrer leurs cours sur la participation des étudiant.e.s et sur le débat... mais qui en réalité 
comme l'a souligné Ollagnier (2014: 70), bien souvent «les étudiants assument le fait que la vérité est objective et universelle et ne veulent pas toujours considérer d'autres manières de penser qui restent marginales ». D'autre part, nous l'avons indiqué, les stagiaires ont déjà construit un rapport à l'institution universitaire ou scolaire qui les emploie et que représente d'une certaine manière la formatrice ou le formateur. En contexte de formation, certaines personnes ont adopté des attitudes qui peuvent sembler passives ou d'opposition, mais qui leur ménagent des possibilités d'autonomie, voire de résistance, au sein de la relation pédagogique. De la même manière que les filles d'école primaire ne semblent pas toujours percevoir immédiatement les bénéfices d'une répartition égalitaire de la parole en classe, mais savent bien reconnaître les risques de perdre l'indépendance qu'elles se sont construites du fait de la moindre attention que les enseignantes et les enseignants ont tendance à leur accorder dans les échanges (Pasquier 2016), les stagiaires constatent que la pédagogie mise en œuvre dans les récits menace leur activité buissonnière et leur possibilité de rester en retrait sans pour autant faire intérieurement preuve de passivité à l'égard de ce qui se passe en classe.

C'est ce passage d'une pédagogie à l'autre qu'il est nécessaire de construire, et le manque de temps pour ce faire semble peut-être conduire certaines formatrices à considérer comme allant de soi, une fois énoncés, les principes sur lesquels repose leur dispositif pédagogique ${ }^{8}$. Une telle démarche inviterait à interroger davantage les rapports sociaux à l'œuvre en contexte de formation qu'ils ne paraissent l'être à lire les récits du corpus, notamment dans une perspective intersectionnelle. Jacqueline Girardat, Émilie Jung et Joëlle Magar-Braeuner (2014 : 236) défendent une telle approche dans le contexte de l'analyse rétrospective d'une formation en travail social au cours de laquelle elles « se sont senties dépassées par les rapports de domination à l'œuvre au sein du groupe ». Elles invitent à mettre à l'épreuve le contexte même de la formation, ce qui s'y passe et s'y dit, en analysant avec les stagiaires les rapports de pouvoir à l'œuvre dans les échanges au cours de la session. Elles préconisent « qu'un premier temps de la formation soit consacré à une réflexion individuelle sur

passent leur temps à poser des questions faussement ouvertes et à attendre des réponses réellement fermées, reproduisant malgré elles.eux un rapport unifocalisé sur l'enseignant.e, et centré de fait sur des savoirs décomposés en "notions". » En même temps qu'est soulignée la difficulté pour des formatrices et des formateurs de se défaire, aussi, d'une forme scolaire traditionnelle, il est possible de se demander si la mise en évidence de l'échec de certaines pratiques ne les amènent pas à adapter leur pédagogie en situation. $\mathrm{Ce}$ faisant, ces personnes oblitèrent d'autant plus la possible réussite de leurs stratégies, qu'elles passent d'un modèle de formation à l'autre, ce qui empêcherait les étudiantes et les étudiants de se construire, très progressivement, de nouveaux repères.

8 Un tel constat pose bien évidemment la question des choix réalisés par l'établissement d'enseignement, notamment en termes d'heures de formation proposées. Ils échappent bien souvent aux formatrices et aux formateurs et les conduisent à prioriser certains objectifs et à faire des choix forcément insatisfaisants. 
UNE MÉTA-ANALYSE DE RÉCITS DE PRATIQUE | 155

la place de chacun et chacune au sein des rapports sociaux de classe, de race, de genre » (ibid. : 248). Elles insistent enfin sur la nécessité d'une meilleure prise en considération de la charge émotionnelle que peut impliquer une telle mise en perspective in situ, pour permettre aux personnes présentes «de désamorcer les postures antagonistes [...] pour favoriser une démarche agoniste, soit la confrontation des points de vue, voire l'émergence de conflits, mais toujours dans l'écoute et sans violence » (ibid.). La difficulté tient alors au risque de démultiplication des objectifs entre nécessité d'un travail sur soi en situation pour déconstruire les rapports sociaux et volonté de former des praticiennes et des praticiens susceptibles de penser le questionnement et la modification de leur pratique, tant dans les domaines de gestion de classe, des démarches pédagogiques et didactiques que dans celui du questionnement critique des savoirs scolaires en contexte d'enseignement (Pasquier 2013a).

\section{RÉFÉRENCES}

ASSOCIATION NATIONALE DES ÉTUDES FÉMINISTES (ANEF)

2014 Le genre dans l'enseignement supérieur et la recherche. Livre blanc. Paris, La Découverte.

AYRAL, Sylvie

2011 La fabrique des garçons. Sanctions et genre au collège. Paris, Presses universitaires de France.

BAUDOUX, Claudine, et Albert NOIRCENT

1997 "L'école et le curriculum caché », dans Laure Gaudreault (dir.), Femmes, éducation et transformations sociales. Montréal, Les éditions du remueménage : 105-128.

BAUTIER, Élisabeth, et Patrick RAYOU

2009 Les inégalités d'apprentissage: programmes, pratiques et malentendus scolaires. Paris, Presses universitaires de France.

BERENI, Laure, et autres

2012 Introduction aux études sur le genre. $2^{\mathrm{e}}$ éd. Bruxelles, De Boeck.

BLAIS, Mireille, et Stéphane MARTINEAU

2006 «L'analyse inductive générale : description d'une démarche visant à donner un sens à des données brutes ", Recherches qualitatives, 26, $2: 1-18$.

BONNERY, Stéphane

2014 «Les albums supports de socialisation masculine et de socialisation lectorale », dans Christiane Connan-Pintado et Gilles Behoteguy (dir.), Etre une fille, un garçon dans la littérature pour la jeunesse. France 1945-2012. Bordeaux, Presses universitaires de Bordeaux : 209-223.

BOUCHARD, Pierrette, et Jean-Claude ST-AMANT

1996 Garçons et filles, stéréotypes et réussite scolaire. Montréal, Les éditions du remue-ménage. 
PASQUIER ET RICHARD | 156

BRESSOUX, Pascal, et Pascal PANSU

2003 Quand les enseignants jugent leurs élèves. Paris, Presses universitaires de France.

DELPHY, Christine

2007 «Le mythe de l'égalité déjà là : un poison! », conférence présentée à l'Institut de recherches et d'études féministes (IREF), Montréal, 11 octobre.

DHUME, Fabrice, Floreal SOTTO et Nora EL MASSIOUI

2015 Former et enseigner sur la (non-)discrimination à l'école? Un enjeu politique incertain. Paris, Alliance de recherche sur les discriminations et le défenseur des droits.

DURU-BELLAT, Marie

1995 «Filles et garçons à l'école, approches sociologiques et psycho-sociales. $2^{\mathrm{e}}$ partie : la construction scolaire des différences entre les sexes ", Revue française de pédagogie, 110, $1: 75-109$.

GIRARDAT, Jacqueline, Émilie JUNG et Joëlle MAGAR-BRAEUNER

2014 «Le concept d'intersectionnalité à l'épreuve de la pratique : l'exemple de la formation Regards croisés sur l'égalité et les discriminations », Nouvelles Pratiques sociales, $262: 235-250$.

HAUT CONSEIL À L'ÉGALITÉ ENTRE LES FEMMES ET LES HOMMES (HCE)

2016 Formation à l'égalité filles-garçons : faire des personnels enseignants et d'éducation les moteurs de l'apprentissage et de l'expérience de l'égalité. Paris, Haut Conseil à l'égalité entre les femmes et les hommes.

LECHENET, Annie, Mireille BAURENS et Isabelle COLLET (dir.)

2016 Former à l'égalité : Défi pour une mixité véritable. Paris, L'Harmattan.

MARRO, Cendrine, Gaël PASQUIER et Laurence BRETON

2016 «Les ressentis émotionnels, une entrée pour éduquer à l'égalité des sexes et à la littérature à l'école primaire », Tréma, 46 : 77-88.

MINISTÈRE DE L'ÉDUCATION NATIONALE

2002 Cahier des charges de la deuxième année de formation dans les instituts universitaires de formation des maîtres (IUFM). Paris, Ministère de l'Éducation nationale.

MOSCONI, Nicole (dir.)

1998 Égalité des sexes en éducation et formation. Paris, Presses universitaires de France.

OLLAGNIER, Edmée

2014 Femmes et défis pour la formation des adultes. Paris, L'Harmattan.

PASQUIER, Gaël

À paraître «Égalité des sexes et EPS : quelles représentations dans les textes officiels de l'Éducation nationale pour quelles situations d'apprentissage? », dans Fabienne Brière-Guenoun et autres (dir.), Les inégalités d'accès aux savoirs se construisent aussi en EPS... Analyses didactiques et sociologiques. Besançon, Presses universitaires de Franche-Comté. 
UNE MÉTA-ANALYSE DE RÉCITS DE PRATIQUE | 157

2016 « Du contrôle de ses actions à l'implication des élèves : la mise en place d'une gestion égalitaire de la prise de parole entre les filles et les garçons par des enseignant-e-s d'école primaire ", dans Annie Léchenet, Mireille Baurens et Isabelle Collet (dir.), Former à l'égalité : défi pour une mixité véritable. Paris, L'Harmattan : 275-287.

2013a «L'enseignement de la langue française à l'école primaire à l'épreuve de la déconstruction du genre », Formation et pratiques d'enseignement en questions, 16 : 161-178.

2013b Les pratiques enseignantes en faveur de l'égalité des sexes et des sexualités. Vers un nouvel élément du curriculum. Thèse de doctorat. Paris, Université Paris Nanterre.

PASQUIER, Gaël, Cendrine MARRO et Laurence BRETON

2016 «Éduquer à l'égalité des sexes à l'école primaire : autour de quelques gestes professionnels accompagnant une activité de lecture littéraire », Le Français aujourd'hui, 193 : 97-107.

PERRENOUD, Philippe

2000 Pédagogie différenciée : des intentions à l'action. Paris, ESF.

RICHARD, Gabrielle

2017 «"Il y a au moins trois gais dans la classe": apports d'une analyse des pratiques enseignantes pour comprendre l'hétéronormativité à l'école ", Nouvelles Pratiques sociales, 281 : 107-124.

2014 Pratiques enseignantes et diversité sexuelle. Analyse des pratiques pédagogiques et d'intervention d'enseignants de l'école secondaire québécoise. Thèse de doctorat. Montréal, Université de Montréal.

SALLE, Muriel

2016 "À l'école de la République, de "l'égalité filles/garçons" à la “ culture de l'égalité " ", Tréma, $46: 5-13$.

TRACHMAN, Mathieu

2014 « Genre : état des lieux. Entretien avec Laure Bereni », dans Laure Bereni et Mathieu Trachman (dir.), Le genre, théories et controverses. Paris, Presses universitaires de France : 11-29.

ZAIDMAN, Claude

1996 La mixité à l'école primaire. Paris, L'Harmattan.

\section{CORPUS ÉTUDIÉ}

BAURENS, Mireille

2008 «Vers une compétence transculturelle du genre dans la formation des enseignant-e-s », Ela. Études de linguistique appliquée, 4, 152 : 429-442.

2005 «L'expérience des IUFM », Les Cahiers du CEDERF - Transmission : savoirs féministes et pratiques pédagogiques, 13: 133-141, [En ligne], [journals.openedition.org/cedref/634] (7 juin 2018). 
PASQUIER ET RICHARD | 158

BAURENS, Mireille, et Caroline SCHREIBER

2010 «Comment troubler les jeunes enseignant $\mathrm{e} \cdot \mathrm{s}$ sur la question du genre à l'école? Analyse d'une expérience de six ans de formation en IUFM », Nouvelles Questions féministes, 2, 29 : 72-87.

BOURDIER-PORHEL, Isabelle

2016 «Construire la mixité et l'égalité filles-garçons au sein d'un collège en éducation prioritaire ", dans Annie Léchenet, Mireille Baurens et Isabelle Collet (dir.), Former à l'égalité : défi pour une mixité véritable. Paris, L'Harmattan : 223-232.

COLLET, Isabelle

2016 «Prendre en compte la violence de genre dans un établissement du secondaire », dans Annie Léchenet, Mireille Baurens et Isabelle Collet (dir.), Former à l'égalité : défi pour une mixité véritable. Paris, L'Harmattan : 289300.

COLLET, Isabelle, et Isabelle GRIN

2013a «En formation initiale des enseignants », Les Cahiers pédagogiques, 487 : 30-31.

2013 b «L'introduction du genre dans la formation initiale des enseignant-e-s : un combat emblématique fait de convictions militantes et de volonté politique ", Formation et pratiques d'enseignement en questions, $16: 31-45$.

COLTICE, Michelle, et Sigolène COUCHOT-SCHIEX

2006 «Regards sur la mixité par un groupe d'enseignants stagiaires en EPS », Revue EPS - La mixité en éducation physique. Paroles, réussites, différenciations, 67 : 122-140.

COSTES, Josette

2014 «Quels contenus donner à la formation des enseignants à l'égalité fillesgarçons », dans Marie Estripeault-Bourjac et Nicolas Sembel (dir.), Femmes, travail, métiers de l'enseignement. Rapports de genre et rapports de classe. Paris, Presses universitaires de Rouen et du Havre : 329-344.

COSTES, Josette, et autres

2015 «Persistance d'inégalités filles/garçons à l'école en France et lien avec la formation des enseignant.e.s », Revue internationale d'ethnographie, $4: 61$ 73.

COUCHOT-SCHIEX, Sigolène

À paraître "Collaborer pour développer l'égalité filles-garçons. Quelle dynamique dans l'académie de Créteil? », Éducation \& Formations.

COUCHOT-SCHIEX, Sigolène, Geneviève COGÉRINO et Michelle COLTICE

2009 « Professeurs stagiaires en EPS face à l'enseignement en mixité », Carrefours de l'éducation, 1, 27 : 169-182.

DAYER, Caroline

2016 «(Ré)agir face aux violences. Vers une éducation et des dispositifs de formation plus égalitaires », dans Annie Léchenet, Mireille Baurens et 
UNE MÉTA-ANALYSE DE RÉCITS DE PRATIQUE | 159

Isabelle Collet (dir.), Former à l'égalité : défi pour une mixité véritable. Paris, L'Harmattan : 301-312.

FONTANINI, Christine

2005 «La formation des enseignantes et des enseignants à l'égalité des chances filles-garçons : une utopie? », Recherches féministes, 18, 1 : 101-115.

GAUTHIEZ-RIEUCAU, Dominique

2016 «Un atelier d'écriture thématique sur la mixité sexuée », dans Annie Léchenet, Mireille Baurens et Isabelle Collet (dir.), Former à l'égalité : défi pour une mixité véritable. Paris, L'Harmattan : 261-272.

GUILPAIN, Geneviève

2016 «La formation continue dans le premier degré : le défi d'un cadre horaire contraint », dans Annie Léchenet, Mireille Baurens et Isabelle Collet (dir.), Former à l'égalité : défi pour une mixité véritable. Paris, L'Harmattan : 209222.

HOUADEC, Virginie

2016 « Les formations des enseignant-es et des personnels de l'éducation à la prise en compte du genre, propositions pour le $1^{\text {er }}$ degré », dans Annie Léchenet, Mireille Baurens et Isabelle Collet (dir.), Former à l'égalité : défi pour une mixité véritable. Paris, L'Harmattan : 197-208.

JACQUEMET, Nicole

2013 «Le mémoire, un moyen pour penser le genre en formation à l'enseignement? », Formation et pratiques d'enseignement en questions, 16 : 77-90.

LAMAMRA, Nadia, et Myriam POSSE

2013 « Des enseignant-e-s sous tension : entre principe d'égalité et système de genre. Expérience d'un enseignement sur le genre à la HEP Lausanne ", Formation et pratiques d'enseignement en questions, $16:$ 63-76.

LAMBERT, Karine

2015 « Pratique, résistance, stratégie », communication présentée à l'occasion des journées d'étude Genre, didactique, formation, École supérieure du professoral et de l'éducation, Université Paris-Est Créteil, 8 et 9 avril.

MONNARD, Muriel, et Anne SGAR

2016 «L'usage genré de l'espace scolaire : l'exemple des espaces interstitiels », dans Annie Léchenet, Mireille Baurens et Isabelle Collet (dir.), Former à l'égalité : défi pour une mixité véritable. Paris, L'Harmattan : 181-194.

MORIN-MESSABEL, Christine, Séverine FERRIÈRE et Muriel SALLE

2012 «L'éducation à l'égalité " filles-garçons " dans la formation des enseignante-s », Recherche et formation, 69 : 47-64.

PETROVIC, Céline

2016 «Enseignements sur le genre : de l'importance du dispositif», dans Annie Léchenet, Mireille Baurens et Isabelle Collet (dir.), Former à l'égalité : défi pour une mixité véritable. Paris, L'Harmattan : 29-40. 
PASQUIER ET RICHARD $\mid 160$

2013a «La formation des enseignants-es et le genre: quelles résistances?», Formation et pratiques d'enseignement en questions, $16: 47-61$.

2013 b «Partage d'expérience sur l'enseignement du genre: un enjeu pour l'égalité? », communication présentée à l'occasion du Congrès international d'actualité de la recherche en éducation et en formation (AREF), Montpellier, Universités Montpellier 2 et 3, 20 août.

2013 c «Quand ça ne va pas de soi pour les enseignants», Les Cahiers pédagogiques, 487 : 32-33.

2006 «Résistances à l'enseignement sur le genre des futur-es professeur-es des écoles - IUFM Alsace », communication présentée à l'occasion du Colloque "Genre et développement: quels enjeux pour la formation? », Bordeaux, Université Bordeaux 3, du 2 au 4 février.

PLATEAU, Nadine

2014 « De l'associatif à l'institutionnel, vers l'intégration de la dimension du genre dans la formation des enseignants », dans Marie Estripeault-Bourjac et Nicolas Sembel (dir.), Femmes, travail, métiers de l'enseignement. Rapports de genre et rapports de classe. Paris, Presses universitaires de Rouen et du Havre : 345-358.

2007 «"Donner une voiture aux garçons et une poupée aux filles, on le fait sans s'en rendre compte et je trouve bien qu'on en ait pris conscience ", Module de sensibilisation à une pédagogie de l'égalité des filles et garçons à l'école ", dans Nathalie Coulon et Geneviève Cresson (dir.), La petite enfance. Entre familles et crèches, entre sexe et genre. Paris, L'Harmattan : 89-108.

PORHEL, Vincent

2016 «Genre, histoire et formation des enseignants : quels enjeux pour quelles pratiques? », dans Annie Léchenet, Mireille Baurens et Isabelle Collet (dir.), Former à l'égalité : défi pour une mixité véritable. Paris, L'Harmattan : 169180.

RICHARD, Gabrielle

À paraître «Troubling Genders, Disturbing Sexualities : The Perilous Territory of the Pedagogy of Discomfort for Teaching LGBTQ Issues ", dans Nelson Rodriguez (dir.), Teaching LGBTQ+ Studies in Education: Theoretical Perspectives. New York, Palgrave Macmillan.

SALLE, Muriel

2014 «Formation des enseignants: les résistances au genre», Travail, genre et sociétés, $1,31: 69-84$.

SAYAC, Nathalie

2016 «L'initiation à la recherche en didactique des mathématiques : une entrée constructive pour promouvoir l'égalité des chances entre filles et garçons à l'école », dans Annie Léchenet, Mireille Baurens et Isabelle Collet (dir.), Former à l'égalité : défi pour une mixité véritable. Paris, L'Harmattan : 5566. 